\title{
Existence Results for Semilinear Functional Differential System with Nonlocal Conditions
}

\section{S.Chandrasekaran}

Abstract: In this paper, sufficient conditions are given for the existence of partial functional differential equations with nonlocal conditions in an abstract space with the help of the fixed point theorems.

Keywords: Mild solutions, Nonlocal conditions, Fixed point theorems.

\section{INTRODUCTION}

In this paper, we discuss the semilinear functional differential equation with nonlocal conditions of the form

$$
\begin{aligned}
x^{\prime}(t) & =A x(t)+f(t, x(t), x(\rho(t))) & \\
x(0) & =\sum_{i=1}^{m} \nu_{i} x\left(t_{i}\right), & \\
x(t) & =\varphi(t), & t \in J=[0, T]
\end{aligned}
$$

where $T>0 ; 0<t_{1}<t_{2}<t_{3}<\cdots<t_{m}<T$ and $v_{i}$ are real

numbers. Let $X$ be a Banach space with the norm $\mathrm{k} \cdot \mathrm{k}$ and the functional $f: J \times X^{2} \rightarrow X ; \rho: J \rightarrow[-r, T]$ are continuous functions. Let $E:=C([-r, T] ; X)$ be the Banach space of continuous functions $x:[-r, T] \rightarrow X$, equipped with the norm,

$$
u(0)=u_{0},
$$

to the following nonlocal evolution equation.

$$
\begin{aligned}
u^{\prime}(t) & =A u(t)+f(t, u(t)), \\
u(0)+g(u) & =u_{0},
\end{aligned}
$$

where $g: C([0, T] ; X) \rightarrow X$ is a continuous function. The equation (1.4)-(1.5) can be applied in physics with better effect than equation (1.2)-(1.3), see [1, 2] and the references therein related to this matter.

In [2], L.Byszewski studied the existence and uniqueness of mild, strong and classical solutions of the nonlocal Cauchy problem for a semilinear evolution equation of the form $\frac{d}{d t} u(t)+A u(t)=f(t, u(t)), \quad t \in\left[t_{0}, t_{0}+a\right]$ $u\left(t_{0}\right)+g\left(t_{1}, \ldots \ldots, t_{p}, u(\cdot)\right)=u_{0}$,

where $0 \leq t_{0}<t_{1}<\ldots . .<t_{p} \leq t_{0}+a, a>0,-A$ is the infinitesimal generator of a $C_{0}$ semigroup on a Banach space $X, u_{0} \in X$ and $f:\left[t_{0}, t_{0}+a\right] \times X \rightarrow X, g:\left[t_{0}, t_{0}+a\right]^{p} \times X \rightarrow X$ are continuous functions using the semigroup theory and the

Banach fixed point theorem.In [3], L.Byszewski and H.Akca studied the existence of mild and classical solutions of $u^{\prime}(t)$ $=A u(t)+f(t, u t)$ $t \in(0, a]$ $u\left(\tau_{\kappa}+0\right)$

$$
\begin{aligned}
& =\quad Q_{k} u\left(\tau_{\kappa}\right)=u\left(\tau_{k}\right)+I_{\kappa}, \\
& +\quad\left(g\left(u_{t 1}, \ldots, u_{t p}\right)\right)(t)=\varphi(t), \\
& \mathrm{k} x \mathrm{k} E=\sup \{\mathrm{k} x(t) \mathrm{k}: t \in[-r, T]\} .
\end{aligned}
$$

The notion of nonlocal conditions has been used to extend the study of the classical initial value evolution equation

$$
u^{\prime}(t)=A u(t)+f(t, u(t)), \quad 0 \leq t \leq T,
$$

Revised Manuscript Received on July 08, 2019.

S.Chandrasekaran Department of Mathematics, Periyar University Constituent College of Arts and Science,Reddipatty-Po-637102 Idappadi Tk Salem -Dt Tamilnadu, INDIA. Email: chandrusavc@gmail.com First

$$
u\left(t_{0}\right)+g(u)=u_{0}, \quad t \in\left[t_{0}, t_{0}+a\right],
$$

where $t_{0}>0, a>0,-A$ is the infinitesimal generator of a compact $C_{0}$-semigroup of operators on a Banach space using Schauder fixed point theorem.

In [4], H. Akca et al., proved the impulsive functional

$$
\begin{array}{cl}
t=6 \quad \tau k, & \text { a nonlocal Cauchy problem for a semilinear functional } \\
\kappa=1,2, \ldots, k, & \text { differential evolution equation } \\
t \in[-r, 0], & u^{\prime}(t)+A u(t)= \\
f\left(t, u(t), u\left(b_{1}(t)\right), \ldots ., u\left(b_{m}(t)\right)\right),
\end{array}
$$


differential equations with nonlocal conditions of the form where $0<t_{1}<\cdots<t_{p} \leq a, p \in \mathrm{N}, A$ and $I_{\kappa}, \kappa=1,2, \ldots, k$ are linear operators acting in a Banach space $E ; f, g$ and $\varphi$ are given functions satisfying some assumptions, $u_{t}(s)=u(t+s)$ for $t \in[0, a], s \in[-r, 0], I_{\kappa} u\left(\tau_{\kappa}\right)=u\left(\tau_{\kappa}+0\right)-u\left(\tau_{\kappa}-0\right)$ and the impulsive moments $\tau_{\kappa}$ are such that $0<\tau_{1}<\cdots<\tau_{\kappa}<\cdots<a$,

$\kappa \in \mathrm{N}$, by using the Banach contraction theorem.

Recently, Under sufficient conditions, Boucherif [5, 6] studied differential inclusions with nonlocal conditions through fixed point theory. The study of nonlocal problems in integro-differential equations have been treated in several works and we refer [7-10] and the references therein. Further, we utilize the technique developed in [11, 12].

Motivated from the above mentioned works. In this paper, we study the existence results for the system (1.1) by means of fixed point theory. The paper is organized as follows: some preliminaries are presented in the section 2 . In section 3, we investigate the existence results of mild solutions for semilinear functional differential system using the Leray-Schauder alternative fixed point theorem and Banach fixed point theorem. Finally in section 4, we give an application for our abstract results.

\section{PRELIMINARIES}

Before proceeding to main result, we shall set forth some preliminaries that will be used in our subsequent discussion.

We shall assume that $\mathrm{A}: \mathrm{D}(\mathrm{A}) \rightarrow \mathrm{X}$ is the infinitesimal generator of a compact analytic semigroup of uniformly bounded linear operators, $(\mathrm{T}(\mathrm{t})) \mathrm{t} \geq 0$, and there exists $\mathrm{M} \geq 1$ such that $\mathrm{kT}(\mathrm{t}) \mathrm{k} \leq \mathrm{M}$ for all $\mathrm{t} \in \mathrm{J}$, (for more details we refer to [12]), and there exists a bounded operator B on

$\mathrm{D}(\mathrm{B})=\mathrm{X}$ given by the formula

$B=\left(I-\sum_{i=1}^{m} \gamma_{i} T\left(t_{i}\right)\right)^{-1}$. This is possible if, for

$\sum_{\text {instance } i=1}^{m}\left|\gamma_{i}\right|<\frac{1}{M}$.

Definition 2.1. A map $\mathrm{f}: \mathrm{J} \times \mathrm{X} \times \mathrm{X} \rightarrow \mathrm{X}$ is said to be L1-Carath`eodory if: $t \rightarrow f(t, x, y)$ is strongly measurable for each $x, y \in X$.

(i) $(x, y) \rightarrow f(t, x, y)$ is continuous for almost all $t \in J$. (ii) for each positive integer $m>0$, there exists $\alpha_{m} \in L^{1}(J$ : $\mathfrak{R}^{+}$) such that

$$
\begin{aligned}
& \sup _{\|x\| \leq m ;\|y\| \leq m}\|f(t, x, y)\| \leq \alpha_{m}(t){ }_{\text {for }} \\
& t \in J \text {,a.e. }
\end{aligned}
$$

Definition 2.2. $x \in E$ is a mild solution of equations (1.1) if

$$
x(t)=\left\{\begin{array}{lr}
\varphi(t), & t \in J_{1}, \\
\sum_{i=1}^{m} \gamma_{i} T(t) B \int_{0}^{t_{i}} T\left(t_{i}-s\right) f(s, x(s), x(\rho(s))) d s & \\
+\int_{0}^{t} T(t-s) f(s, x(s), x(\rho(s))) d s, & t \in J,
\end{array}\right.
$$

is satisfied.

Our existence theorem is based on the following theorem.

Theorem 2.1. Let $S$ be a convex subset of a Banach space $E$ and assume that $0 \in S$. Let $F: S \rightarrow S$ be a completely continuous operator and let

$U(F)=\{x \in S: x=\lambda F x \quad$ for some $0<\lambda<1\}$.

Then either is $U(F)$ unbounded or $F$ has a fixed point.

\section{EXISTENCE OF A SOLUTION :}

In this section, we prove the existence theorem by using the following hypotheses:

$\left(H_{1}\right)$ : There exists a continuous non-decreasing function for

$$
\Omega: \mathfrak{R}^{+} \rightarrow(0, \infty) \text { and } p \in L^{1}\left(J ; \mathfrak{R}^{+}\right) \text {such that }
$$

$\mathrm{k} f(t, x, y) \mathrm{k} \leq p(t) \Omega(\mathrm{k} x \mathrm{k}+\mathrm{k} y \mathrm{k}), \quad t \in J ; x, y \in X$.

$\left(H_{2}\right)$ : The function $\rho: J \rightarrow[-r, T]$ is continuous and $t-r \leq \rho(t)$

$$
\leq t \text {, for every } t \in J \text {. }
$$

Theorem 3.1. If the hypotheses $\left(H_{1}\right)-\left(H_{2}\right)$ be hold. Then the system (1.1) has a mild solution $x(t)$ on $[-r, T]$ provided that following inequality is satisfied: 
$\sup _{\varpi \in[0, \infty)} \frac{\varpi}{M\|p\|_{L^{1}} \Omega(2 \varpi)\left[1+M\|B\| \sum_{i=1}^{m}\left|\gamma_{i}\right|\right]}>1$

Proof. Let $T$ be an arbitrary number $0<T<+\infty$ satisfying

(3.1). It follows from (3.1) that there exists $\beta>0$ such that

$$
\frac{\beta}{M\|p\|_{L^{1}} \Omega(2 \beta)\left[1+M\|B\| \sum_{i=1}^{m}\left|\gamma_{i}\right|\right]}>
$$

Step-1:

For $\lambda \in(0,1)$, let consider the problems

$$
\begin{aligned}
x(t)= & \lambda \sum_{i=1}^{m} \gamma_{i} T(t) B \int_{0}^{t_{i}} T\left(t_{i}-s\right) f(s, x(s), x(\rho(s))) d s \\
& +\lambda \int_{0}^{t} T(t-s) f(s, x(s), x(\rho(s))) d s, t \in J .
\end{aligned}
$$

Notice that if $x \in E$ is a solution of (3.3) for $\lambda=1$, then $x$ is a solution of (1.1).

Consider $U=\{x \in E ; \mathrm{k} x \mathrm{k}<\beta\}$. Define $F: U^{-} \rightarrow E$ by

$$
\begin{aligned}
F x(t) & =\sum_{i=1}^{m} \gamma_{i} T(t) B \int_{0}^{t_{i}} T\left(t_{i}-s\right) f(s, x(s), x(\rho(s))) d s \\
& +\int_{0}^{t} T(t-s) f(s, x(s), x(\rho(s))) d s \quad t \in J,
\end{aligned}
$$

we can easily show that $F$ is continuous.

Step-2 : $F$ maps bounded sets into bounded sets.

For, let $x \in B_{\rho}=\left\{v \in E: \mathrm{k} v \mathrm{k} \leq_{+}\right\}$, then $\left(H_{1}\right)-\left(H_{2}\right)$ implies that

$$
\begin{aligned}
\|F x(t)\|= & \left\|\sum_{i=1}^{m} \gamma_{i} T(t) B \int_{0}^{t_{i}} T\left(t_{i}-s\right) f(s, x(s), x(\rho(s))) d s\right\| \\
& +\left\|\int_{0}^{t} T(t-s) f(s, x(s), x(\rho(s))) d s\right\| \\
\leq & M^{2}\|B\| \sum_{i=1}^{m}\left|\gamma_{i}\right| \int_{0}^{t_{i}}\|f(s, x(s), x(\rho(s))) d s\| \\
& +M \int_{0}^{t}\|f(s, x(s), x(\rho(s))) d s\| \\
& +M \int_{0}^{t} p(s) \Omega(\|x(s)\|+\|x(\rho(s))\|) d s, \\
\leq & M^{2}\|B\| \sum_{i=1}^{m}\left|\gamma_{i}\right| \int_{0}^{t_{i}} p(s) \Omega(2 \varpi) d s+M \int_{0}^{t} p(s) \Omega(2 \varpi)
\end{aligned}
$$

Since $T(t)$ is compact for every $t>0$, the set $\left\{F_{Q} x(t): x \in U^{-}\right\}$is precompact in $X$. for

$(0, t)$. Moreover for $x \in U^{-}$we have $t$

$U^{-}=$ every $q \in$ $\mathrm{Z}$

$$
\begin{gathered}
F_{Q X}(t)-F x(t) \mathrm{k} \leq \mathrm{k} T(t \\
-s) f(s, x(s), x(\rho(s))) \mathrm{k} d s \\
t-Q \\
t M \alpha_{\beta}(s) d s . \\
t-Q \rightarrow 0 \text { as } Q \rightarrow 0 .
\end{gathered}
$$

Since $\alpha_{\beta}(s) \in L^{1}$ and meas $([t-q, t])<Q$.

Step-3: $\quad F\left(U^{-}\right)$is a uniformly equicontinuous family of

For, let $\tau_{1}<\tau_{2}$ in $J$. Then

Since, see [13, Proposition 1] and [6] that there exists $\eta>0$ such that

as $\tau_{2} \rightarrow \tau_{1}$ we get $\mathrm{k} T\left(\tau_{1}\right)-T\left(\tau_{2}\right) \mathrm{k} \rightarrow 0, \operatorname{maxk} T\left(\tau_{1}-s\right)-T\left(\tau_{2}-s\right) \mathrm{k}$

$\int_{\tau_{1}}^{\tau_{2}} \alpha_{\beta}(s) d s \rightarrow 0$ as $\tau_{2} \rightarrow \tau_{1}^{s \in J}$. Because $\alpha_{\beta} \in L^{1}\left({ }^{J} \mathfrak{R}^{+}\right)$.

Step- 4: The set ${ }^{U_{-}}(t)=\left\{F x(t): x \in^{U_{-}}\right\}$is precompact in $E$.

For, let $t>0$ and $0<q<t$. For $x \in U_{-}$define

$$
\begin{aligned}
F_{\epsilon} x(t) & =\sum_{i=1}^{m} \gamma_{i} T(t) B \int_{0}^{t_{i}} T\left(t_{i}-s\right) f(s, x(s), x(\rho(s))) d s \\
& +\int_{0}^{t-\epsilon} T(t-s) f(s, x(s), x(\rho(s))) d s
\end{aligned}
$$

Step- 5:

Next, by $\left(H_{1}\right)$ and $\left(H_{2}\right)$ all solutions of (3.3) satisfy

so that, 
$\|x\| \leq M\left[M\|B\| \sum_{i=1}^{m}\left|\gamma_{i}\right|+1\right]\|p\|_{L^{1}} \Omega(2\|x\|)$

If $t \in J_{1}$, then $\mathrm{k} x(t) \mathrm{k}=\mathrm{k} \phi \mathrm{k}$ and the previous inequality holds. Consequently,

$$
\begin{aligned}
\|x(t)\| \leq & \left\|\sum_{i=1}^{m} \gamma_{i} T(t) B \int_{0}^{t_{i}} T\left(t_{i}-s\right) f(s, x(s), x(\rho(s))) d s\right\| \\
& +\left\|\int_{0}^{t} T(t-s) f(s, x(s), x(\rho(s))) d s\right\| \\
\leq & M^{2}\|B\| \sum_{i=1}^{m}\left|\gamma_{i}\right| \int_{0}^{t_{i}}\|f(s, x(s), x(\rho(s))) d s\|+M \int_{0}^{t}\|f(s, x(s), x(\rho(s))) d s\| \\
\leq & M\left[M\|B\| \sum_{i=1}^{m}\left|\gamma_{i}\right|+1\right]\|\ell\|_{L^{1}}+M^{2}\|B\| \sum_{i=1}^{m}\left|\gamma_{i}\right| \int_{0}^{t_{i}} \ell(s)(\|x(s)\|+\|x(\rho(s))\|) d s \\
\Omega(2 \| & \left.x \|_{E}+\mathbb{N}\right) \int_{0}^{t} \ell(s)(\|x(s)\|+\|x(\rho(s))\|) \| d s, \\
\leq & M\left[M\|B\| \sum_{i=1}^{m}\left|\gamma_{i}\right|+1\right]\|\ell\|_{L^{1}}+2 M^{2}\|B\| \sum_{i=1}^{m}\left|\gamma_{i}\right| \int_{0}^{t_{i}} \ell(s)\|x(s)\| d s \\
& +2 M \int_{0}^{t} \ell(s)\|x(s)\| d s, \\
\leq & M\left[M\|B\| \sum_{i=1}^{m}\left|\gamma_{i}\right|+1\right]\|\ell\|_{L^{1}}+2 M\left[M\|B\| \sum_{i=1}^{m}\left|\gamma_{i}\right|+1\right] \int_{0}^{t} \ell(s)\|x(s)\| d s .
\end{aligned}
$$$$
\|x\|_{E} \leq M\left[M\|B\| \sum_{i=1}^{m}\left|\gamma_{i}\right|+1\right]\|p\|_{L^{1}} \Omega\left(2\|x\|_{E}^{+N}\right) \int_{0}^{t} \ell(s)(\|x(s)\|+\|x(\rho(s))\|) d s,
$$

that $x \in \lambda F x$. Then $x$ satisfies (3.3) and $\mathrm{k} x \mathrm{k} E=\beta$. It follows

from (3.4) that

$$
\beta \leq M\left[M\|B\| \sum_{i=1}^{m}\left|\gamma_{i}\right|+1\right]\|p\|_{L^{1}} \Omega(2 \beta)
$$

$$
\|x(t)\| \leq Q_{1}+Q_{2} \int_{0}^{t} \ell(s)\|x(s)\| d s,
$$

$$
Q_{1}=M\left[M\|B\| \sum_{i=1}^{m}\left|h_{i}\right|+1\right]\|\ell\|_{L^{r}} \text { and } Q_{2}=2 M\left[M\|B\| \sum_{i=1}^{m}\left|\gamma_{i}\right|+1\right]
$$

Thuse.If $t \in J_{1}$, then $\mathrm{k} x(t) \mathrm{k}=\mathrm{k} \phi \mathrm{k}$ and the previous inequality holds.By applying Gronwall inequality,

$$
\|x\|_{E} \leq Q_{1} \exp \left(Q_{2}\|\ell\|_{L^{1}}\right), t \in J,
$$

(3.2)). Moreover, the set $U$ is bounded. Consequently, by Theorem 2.1, the operator $F$ has a fixed point in $E$.

Therefore, the system (1.1) has a mild solution. Thus the proof is completed.

We now present another existence result for system (1.1). The Lipschitz condition on $f$ is relaxed by using Wintner growth condition in the following Theorem.

Theorem 3.2. Assume that $\left(\mathrm{H}_{2}\right)$ and the following condition holds

$\left(H_{3}\right)$ : There exists $\ell \in L^{1}\left([0, T], \Re^{+}\right)$such that $\mathrm{k} f\left(t, x_{1}, y_{1}\right)$ $-f\left(t, x_{2}, y_{2}\right) \mathrm{k} \leq \ell(t) \mathrm{hk} x_{1}-x_{2} \mathrm{k}+\mathrm{k} y_{1}-y_{2} \mathrm{ki}, x_{i}, y_{i} \in X$

$$
\text { and } \quad \mathrm{k} f(t, 0,0) \mathrm{k} \quad \leq \ell(t) \text {, a.e. } t \in J \text {, }
$$

then the system (1.1) has at least one mild solution on $[-r, T]$.Proof. The operator $F$ defined in the proof of the previous theorem is completely continuous. Now, we prove that $U=\{x \in E: x \in \lambda F(x)$ for some $\lambda \in(0,1)\}$ is bounded.

$$
\begin{aligned}
& \text { Let } \quad \mathbf{x} \in \mathbf{U} \text {. Then for each } \mathbf{t} \in \\
& \mathbf{J} \\
& x(t)=\lambda \sum_{i=1}^{m} \gamma_{i} T(t) B \int_{0}^{t_{i}} T\left(t_{i}-s\right) f(s, x(s), x(\rho(s))) d s+\lambda \int_{0}^{t} T(t-s) f(s, x(s), x(\rho(s))) d s, \\
& \text { or some } \lambda \in(0,1) \text {. Then }
\end{aligned}
$$

\section{$\|x\|_{E} \leq \beta_{1}$.}

This shows that the set $U$ is bounded $\square$ As a consequence of Theorem 2.1, we deduce that $F$ has a fixed point which is a mild solution of (1.1). This completes the proof. Concerning the existence and uniqueness of mild solution for the system (1.1), we establish in the following result.

Theorem 3.3. Let assumption $\left(\mathrm{H}_{2}\right)$ be verified and the following condition holds $\left(H_{4}\right)$ : There exists constants $\ell_{1}>0$ such that $\mathrm{k} f\left(t, x_{1}, y_{1}\right)$ $-f\left(t, x_{2}, y_{2}\right) \mathrm{k} \leq \ell_{1}\left(\mathrm{k} x_{1}-x_{2} \mathrm{k}+\mathrm{k} y_{1}-y_{2} \mathrm{k}\right), x_{i}, y_{i} \in X$.If $\Lambda=2 M\left[M\|B\| \sum_{i=1}^{m}\left|\gamma_{i}\right|+1\right] \ell_{1}<1$ then there exists a unique mild solution for the system (1.1).Proof. The operator $F$ defined as in the proof of the previous theorem. Now, we shall show that the operator $F$ is a contraction. Let $x \in$ $U$, then for each $t \in[-r, T]$ we have

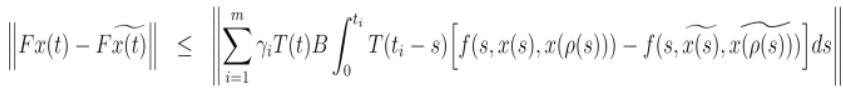

$$
\begin{aligned}
& +\| \int_{0}^{t} T(t-s)[f(s, x(s), x(\rho(s)))-f(s, \widetilde{x(s)}, \widetilde{x(\rho(s)))]}] d s \|
\end{aligned}
$$

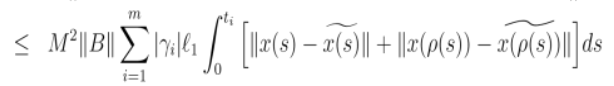

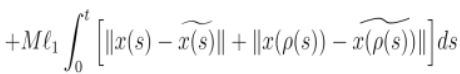

$$
\begin{aligned}
& \leq 2 M\left[M\|B\| \sum_{i=1}^{m}\left|\gamma_{i}\right|+1\right] \ell_{1} \int_{0}^{t}\|x(s)-\widetilde{x(s)}\| d s .
\end{aligned}
$$


Taking supremum over $t \in[-r, T]$, we get,

$$
\|F x-F \widetilde{x}\|_{E} \leq 2 M\left[M\|B\| \sum_{i=1}^{m}\left|\gamma_{i}\right|+1\right] \ell_{1}\|x-\tilde{x}\|_{E}
$$

Thus,

$\mathrm{k} F x-F x \mathrm{ek} E \leq \Lambda \mathrm{k} x-x \mathrm{ek}$, (3.5)

since $0<\Lambda<1$. This shows that operator $F$ is a contraction. Uniqueness follows from $\left(H_{4}\right)$. Consequently, by (3.5), the operator $F$ satisfies all the assumptions of the Banach fixed point theorem. Therefore, in space $U$ there is only one fixed point of $F$ and this is the mild solution of the system (1.1). So, the proof of Theorem 3.3 is complete.

\section{CONCLUSION}

In this section, we give an example of the partial differential equation to illustrate the application of our main theorem

$$
\begin{aligned}
& \frac{\partial v(t, u)}{\partial t}=\frac{\partial^{2} v(t, u)}{\partial u^{2}}+\mu(t, u, v(t, u), v(\rho(t), u)) \\
& v(t, 0)=v(t, \pi)=0, \quad t \in J=[0,1], u \in I=[0, \pi \\
& v(0, u)=\sum_{i=1}^{n} \alpha_{i} v\left(t_{i}, u\right), \quad u \in I \\
& v(t, u)=\varphi(t, u) \text { for }-r \leq t \leq 0 \\
&]
\end{aligned}
$$

where $\mu: \mathrm{J} \times \mathrm{I} \times \mathrm{X} \times \mathrm{X} \rightarrow \mathrm{X} ; \rho: \mathrm{J} \rightarrow[-\mathrm{r}, 1]$ are continuous and $\mathrm{t}-\mathrm{r} \leq \rho(\mathrm{t}) \leq \mathrm{t}$ for every $\mathrm{t} \geq 0$ and $\mathrm{ti} \in \mathrm{J} ; \alpha \mathrm{i} \in \mathfrak{R}$ are prefixed numbers. Let $\mathrm{X}=\mathrm{L} 2[0, \pi]$. Define $\mathrm{A}$

an operator on $X$ by $A v=\frac{\partial^{2} v}{\partial u^{2}}$ with the domain

$$
D(A)=\left\{v \in X \mid v \text { and } \frac{\partial v}{\partial u}\right. \text { are absolutely }
$$

$$
\text { continuous, } \left.\frac{\partial^{2} v}{\partial u^{2}} \in X, v(0)=v(\pi)=0 .\right\}
$$

It is well known that generates a strongly continuous semigroup $\mathrm{T}(\mathrm{t})$ which is compact, analytic and self adjoint. Moreover, the operator A can be expressed as

$$
A u=\sum_{n=1}^{\infty} n^{2}<v, v_{n}>v_{n}, \quad v \in D(A)
$$

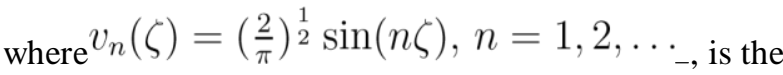
orthonormal set of eigenvectors of $\mathrm{A}$.

Then the operator $(-A)^{\frac{1}{2}}$ is given by

$$
\begin{aligned}
(-A)^{\frac{1}{2}} v & =\sum_{n=1}^{\infty} n<v, v_{n}>v_{n} \\
D\left[(-A)^{\frac{1}{2}}\right] & =\left\{v \in X ; \sum_{n=1}^{\infty} n<v, v_{n}>v_{n} \in X\right\}
\end{aligned}
$$

This satisfies $\mathrm{kT}(\mathrm{t}) \mathrm{k} \leq 1, \mathrm{t} \geq 0$, and hence is a contraction semigroup. In particular,

$$
\left\|(-A)^{-\frac{1}{2}}\right\|=\frac{1}{\Gamma \frac{1}{2}} \int_{0}^{\infty} t^{\frac{1}{2}-1}\|T(t)\| d t \leq 1
$$

.The problem (4.1) can be modeled as the abstract semilinear differential system (1.1).

By defining the operator $\mathrm{f}$ by $\mathrm{f}(\mathrm{t}, \mathrm{x}, \mathrm{y}) \mathrm{u}=\mu(\mathrm{t}, \mathrm{u}, \mathrm{x}(\mathrm{u}), \mathrm{y}(\mathrm{u}))$.

The next result a consequence of Theorem 3.1.

Proposition 4.1. Assume that the hypotheses (H1)-(H2) hold. Then there exists a mild solution $\mathrm{v}$ of the system (4.1) provided

$$
\sup _{\varpi \in[0, \infty)} \frac{\varpi}{\|p\|_{L^{1}} \Omega(2 \varpi)\left[1+\|B\| \sum_{i=1}^{m}\left|\gamma_{i}\right|\right]}>1
$$

is satisfied.

\section{REFERENCES}

1. K. Deng, Exponential decay of solutions of semilinear parabolic equations with nonlocal initial conditions, J. Math. Anal. Appl. 179 (1993) 630-637.

2. L. Byszewski, Theorems about the existence and uniqueness of solutions of a semilinear evolution nonlocal Cauchy problem, J. Math. Anal. Appl. 162 (1991) 494-505.

3. L.Byszewski, H.Acka Existence of solutions of a semilinear functional differential evolution nonlocal problem, Nonlinear Anal. 34(1998) 65-72.

4. Akca Haydar, Boucherif Abdelkadar, Valery Covachev, Impulsive functional differential equations with nonlocal conditions, Int. J. Math. Math. Sci. 29 (5)(2002) 251-256.

5. A. Boucherif, Nonlocal Cauchy problems for first order multivalued differential equations, E. J. Differential Equations, 2002:47 (2002), 1-9.

6. A. Boucherif, Semilinear evolution inclusions with nonlocal conditions, Appl.

7. Math. Lett. 22 (2009) $1145-1149$.

8. K. Balachandran and K. Uchiyama, Existence of solutions of nonlinear integrodifferential equations of sobolev type with nonlocal conditions in Banach spaces, Proc. Indian Acad. Sci. 2(2000) 225- 232

9. K. Balachandran and J.P. Dauer, Existence of solutions for an integrodifferential equations with nonlocal conditions in Banach spaces, Libertas Math. 16 (1996) 133 - 143.

10. Y.Lin, H.Liu, Semilinear integrodifferential equations with nonlocal Cauchy problem, Nonlinear Anal.Theory. Methods.Appl. 26(1996) 1023-1033.

11. H. L. Tidke and M.B. Dhakne, Existence of solutions and controllability of nonlinear mixed integro differential equations with nonlocal condition, AMEN 11 3(2011) 12-22.

12. E. Herna'ndez, S.M. Tanaka Aki and H. Henriquez, Global solutions for impulsive abstract partial differential equations, Comp. Math. Appl 56(2008) 1206-1215.

13. A. Pazy, Semigroups of Linear Operators and Applications to Partial Differential Equations, Springer-Verlag, Newyork, 1983.

14. J.Hofbauer, P.L. Simon, An existence theorem for parabolic equations on $\mathfrak{R N}$ with discontinuous nonlinearity, Electron. J. Qual. Theory Differ. Equ. (8)(2001) 1-9.

\section{AUTHORS PROFILE}

S.Chandrasekaran Department of Mathematics, Periyar University Constituent College of Arts and Science,Reddipatty-Po-637102 Idappadi Tk Salem -Dt Tamilnadu, INDIA. Email: chandrusavc@gmail.com 